\title{
The Use of Combined Three-Dimensional Electron Back Scatter Diffraction/Energy Dispersive X-Ray Analysis to Assess the Characteristics of the Gamma/Gamma-Prime Microstructure in Alloy $720 \mathrm{Li}^{\mathrm{TM}}$
}

\author{
D.J. Child*, G.D. West, R.C. Thomson \\ Department of Materials, Loughborough University, Loughborough, Leicestershire, LE11 3TU, \\ United Kingdom.
}

The nickel-based superalloy Alloy $720 \mathrm{Li}^{\mathrm{TM}}$ consists of a face-centred cubic $\gamma$ matrix with primary $\gamma^{\prime}$ residing at grain boundaries. Quantitative analyses of $\gamma / \gamma^{\prime}$ distributions have previously been limited to two dimensions, introducing a number of geometrical assumptions for three-dimensional estimation. The reconstruction of $\gamma / \gamma^{\prime}$ structures in three dimensions has been demonstrated, which allow observations of morphological aspects such as true particle shape and precipitate phase connectivity $[1,2]$. However, such examples have only produced small scale reconstructions of cuboidal secondary $\gamma^{\prime}$ structures within volumes of $10 \times 10 \times 5 \mu \mathrm{m}$ approximate dimensions. Atom probe techniques are also available, but are again suited to the study of small volumes and quantity.

In this study, data collection has been carried out by employing a serial milling technique with simultaneous electron back scatter diffraction (EBSD) and energy dispersive x-ray (EDX) analysis. Combining EBSD data with EDX is critical in obtaining maps to distinguish between the chemically differing, but crystallographically similar $\gamma$ and $\gamma^{\prime}$ phases present in the alloy studied. EDX is shown to allow the differentiation of $\gamma$ and $\gamma^{\prime}$ phases (FIG 1), whilst EBSD provides grain shape accuracy and allows the determination of interconnected regions. The study identifies an area of grain banding within the alloy, which provides the basis for a three-dimensional comparison of $\gamma^{\prime}$ precipitate size between coarse and fine grain areas (FIG 2). The occurrence of fine grain bands may reduce creep and fatigue crack growth resistance properties in these areas, due to alteration of the $\gamma / \gamma^{\prime}$ distribution $[3,4]$. Identification and characterisation of such features are therefore advantageous in understanding the effects of banding on $\gamma / \gamma^{\prime}$ morphologies.

The analysis also identified coherent $\gamma^{\prime}$ phase particles (FIG 3), possible only using both EDX and EBSD systems simultaneously. The majority of $\gamma^{\prime}$ particles lie in the range of 1-10 $\mu \mathrm{m}$, with a detectable particle size limit of $500 \mathrm{~nm}$ being established. The validity of the reconstruction has been demonstrated using an electron interaction volume model, confirming $\gamma^{\prime}$ phase connectivity in all dimensions.

References

[1] A.C. Lund, P.W. Voorhees, Acta Mat. 50 (2002) 2585-2598.

[2] J. MacSleyne et al., Acta Mat. 57 (2009) 6251-6267.

[3] R.J. Mitchell et al., Proc. of the 11th Int. Symp. on Superalloys (2008) 347-356.

[4] D.A. Woodford, Energy Materials. 1 (2006) 59-79. 


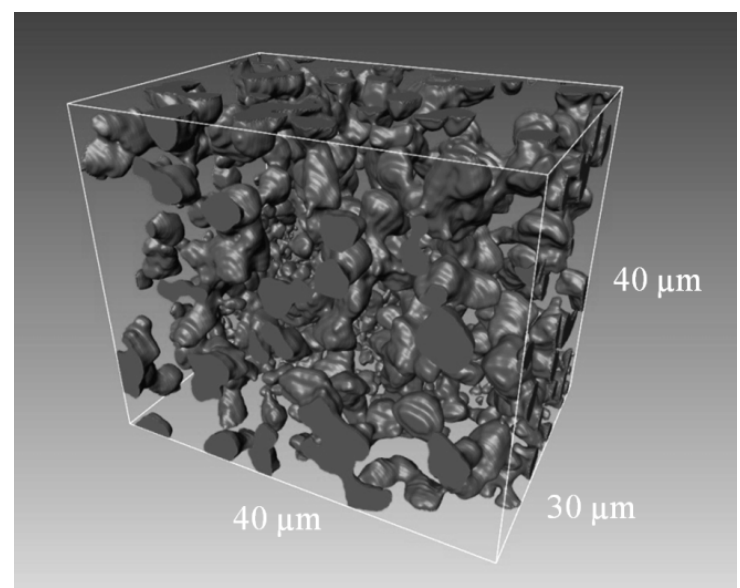

FIG 1. Three-dimensional reconstruction of $\gamma^{\prime}$ phase segmented from consecutive chromium energy dispersive x-ray maps, from a section of Alloy $720 \mathrm{Li}$, using a serial milling technique.

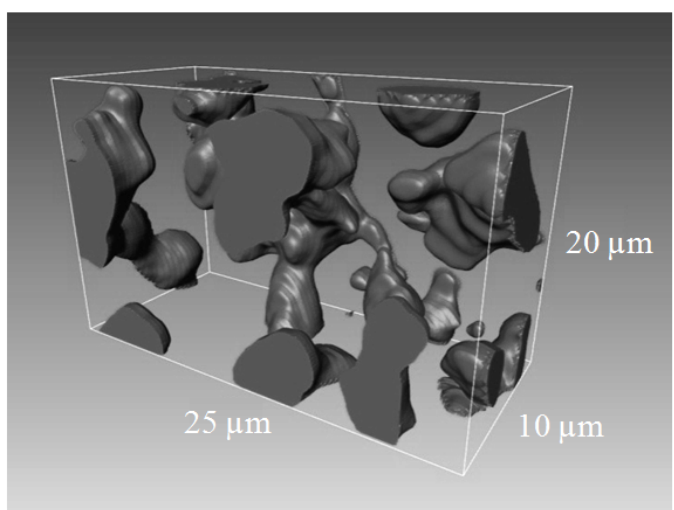

(a)

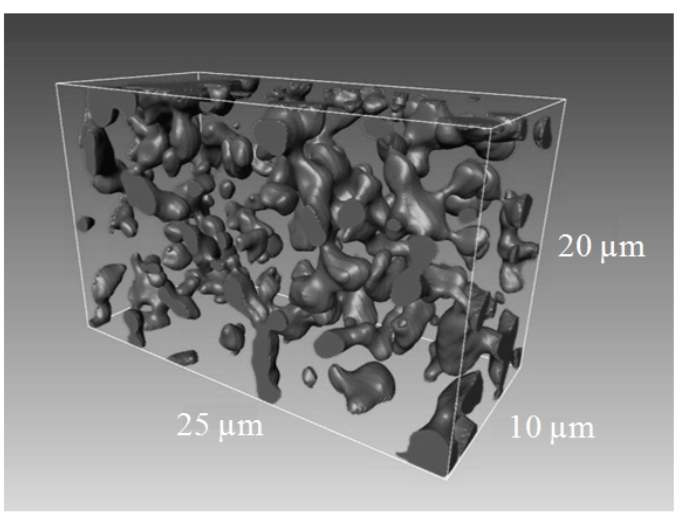

(b)

FIG 2. Three-dimensional reconstruction of $\gamma^{\prime}$ phase segmented from chromium energy dispersive $\mathrm{x}$-ray maps, from a section of Alloy $720 \mathrm{Li}$, showing a: (a) coarse grain region; (b) fine grain region.

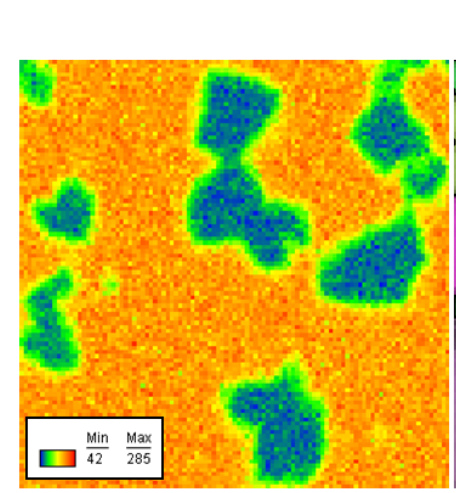

(a)

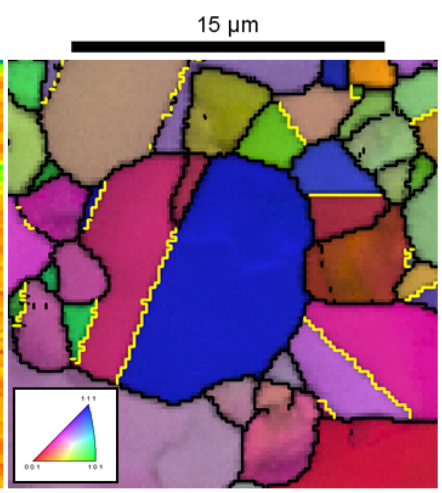

(b)

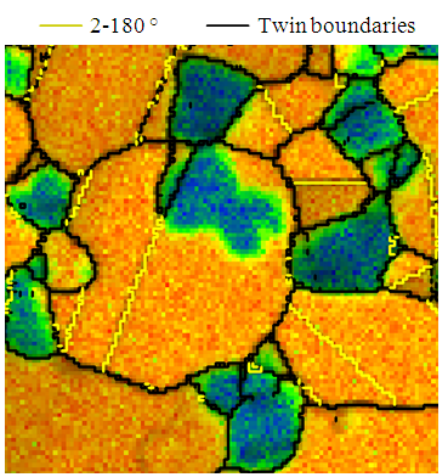

(c)

FIG 3. Data maps of Alloy 720Li, demonstrating the presence of coherent $\gamma$ ', with maps: (a) colour chromium EDX; (b) inverse pole figure + image quality with grain boundary identification; (c) colour chromium EDX + image quality with grain boundary identification. 